\title{
THE EFFECT OF DUST GEOMETRY ON THE Ly $\alpha$ OUTPUT OF GALAXIES
}

\author{
C. Scarlata ${ }^{1}$, J. Colbert ${ }^{1}$, H. I. Teplitz ${ }^{1}$, N. Panagia ${ }^{2,3,4}$, M. Hayes ${ }^{5}$, B. Siana ${ }^{6}$, A. Rau ${ }^{6,7}$, P. Francis $^{8}$, A. Caon ${ }^{1,9}$, \\ A. Pizzella ${ }^{9}$, AND C. BRIDGE ${ }^{1}$ \\ ${ }^{1}$ Spitzer Science Center, California Institute of Technology, 314-6, Pasadena, CA-91125, USA \\ ${ }^{2}$ Space Telescope Science Institute, 3700 San Martin Drive, Baltimore, MD 21218, USA \\ ${ }^{3}$ INAF/Osservatorio Astrofisico di Catania, Via S.Sofia 78, I-95123 Catania, Italy \\ ${ }^{4}$ Supernova Ltd., Olde Yard Village 131, Northsound Road, Virgin Gorda, British Virgin Islands, UK \\ ${ }^{5}$ Observatoire de Geneve, 51, Ch. des Maillettes, CH-1290, Sauverny, Switzerland \\ ${ }^{6}$ California Institute of Technology, MS 105-24, Pasadena, CA 91125, USA \\ ${ }^{7}$ Max-Planck-Institut für extraterrestrische Physik, Giessenbachstrasse 1, 85748 Garching, Germany \\ ${ }^{8}$ Research School of Astronomy and Astrophysics, the Australian National University, Canberra 0200, Australia \\ ${ }^{9}$ Department of Astronomy, University of Padova, Vicolo dell'Osservatorio 3, I-35122, Padova, Italy \\ Received 2009 July 16; accepted 2009 September 8; published 2009 October 5
}

\begin{abstract}
We present the optical spectroscopic follow-up of $31 z=0.3$ Ly $\alpha$ emitters, previously identified by Deharveng et al. We find that $17 \%$ of the Ly $\alpha$ emitters have line ratios that require the hard ionizing continuum produced by an active galactic nucleus. The uniform dust screen geometry traditionally used in studies similar to ours is not able to simultaneously reproduce the observed high $\mathrm{Ly} \alpha / \mathrm{H} \alpha$ and $\mathrm{H} \alpha / \mathrm{H} \beta$ line ratios. We consider different possibilities for the geometry of the dust around the emitting sources. We find that also a uniform mixture of sources and dust does not reproduce the observed line ratios. Instead, these are well reproduced by a clumpy dust screen. This more realistic treatment of the geometry results in extinction corrected $(\mathrm{Ly} \alpha / \mathrm{H} \alpha)_{C}$ values consistent with case B recombination theory, whereas a uniform dust screen model would imply values $(\operatorname{Ly} \alpha / \mathrm{H} \alpha)_{C}$ higher than 8.7. Our analysis shows that there is no need to invoke ad hoc multiphase media in which the Ly $\alpha$ photons only scatter between the dusty clouds and eventually escape.
\end{abstract}

Key words: galaxies: ISM - ISM: structure

\section{INTRODUCTION}

In the pre-James Webb Space Telescope (JWST) era, we depend strongly on the observation of Ly $\alpha$ emission for both spectroscopic confirmation and, often, the actual discovery of very high redshift $(z>6)$ galaxies. Furthermore, the evolution with redshift of the clustering properties and luminosity function of Ly $\alpha$ emitters could be used to infer important information about the re-ionization epoch, if the physics governing the Ly $\alpha$ escape were well understood. In the local universe, where the physical properties of galaxies can in principle be determined in great detail, the number of known Ly $\alpha$ emitting galaxies is extremely small (on the order of a dozen objects; e.g., Giavalisco et al. 1996; Ostlin et al. 2009).

Numerous theoretical works demonstrate how the observed Ly $\alpha$ emission and its equivalent width (EW) depend on different factors including not only the age of the stellar population, the stellar initial mass function, the metal and dust content (Charlot \& Fall 1993), but also the relative geometries of interstellar $\mathrm{HI}$ and $\mathrm{H}$ II regions and the kinematics of the neutral gas (e.g., Panagia \& Ranieri 1973a, 1973b; Neufeld 1990; Verhamme et al. 2006; Dijkstra et al. 2006; Laursen \& Sommer-Larsen 2007). All of these studies point to a preferential attenuation at resonant frequencies, although it is likely that the homogeneous interstellar medium (ISM) model is a great oversimplification. In contrast to the homogeneous case, a multiphase ISM in which the dust lies in cold neutral clouds (which Ly $\alpha$ photons cannot penetrate) would actually serve to preserve Ly $\alpha$ photons, leading to a relative enhancement of the Ly $\alpha \mathrm{EW}$ and the $\mathrm{Ly} \alpha /$ Balmer line ratios (e.g., Neufeld 1991; Hansen et al. 2006). This latter mechanism has been proposed to explain the high-Ly $\alpha$ EW sources observed at high redshift (Finkelstein et al. 2008) and the high-Ly $\alpha / \mathrm{H} \alpha$ ratio inferred for low- $z$ dusty $\operatorname{Ly} \alpha$ emitters (Atek et al. 2008, 2009).

Recently, Deharveng et al. (2008) presented the results from a slitless spectroscopic survey tuned to identify low- $z$ Ly $\alpha$ emitters with the Galaxy Evolution Explorer (GALEX) satellite. The survey covered $\sim 5.6 \mathrm{deg}^{2}$ and identified 96 Ly $\alpha$ emitters with redshifts in the range $0.2<z<0.4$. All galaxies have measured Ly $\alpha$ EW and line intensities. The EW distribution of the $z=0.3$ Ly $\alpha$ emitters is similar to that at $z \sim 3$, but their fraction among star-forming galaxies is smaller.

We are conducting a program of spectroscopic follow-up of the GALEX-Ly $\alpha$ emitters. In this Letter, we present the comparison of the $\mathrm{Ly} \alpha / \mathrm{H} \alpha$ and $\mathrm{H} \alpha / \mathrm{H} \beta$ ratios for the galaxies observed so far.

\section{OBSERVATIONS AND DATA ANALYSIS}

Optical spectra of 31 of the Deharveng et al. $z \sim 0.3$ galaxies were acquired with the Double-Beam Spectrograph (DBSP; Oke \& Gunn 1982) mounted on the Hale $5 \mathrm{~m}$ telescope at Palomar Observatory. The objects were selected to be visible from the Palomar Observatory (in the following fields GROTH, NGPDWS, and SIRTFFL from Deharveng et al. 2008), and to have $z \lesssim 0.32$, so that $\mathrm{H} \alpha$ would fall in the covered spectral range. The sample is presented in Table 1 . We used the D55 dichroic to split the light at $\sim 5500 \AA$ into a blue and a red channel. The red spectra were acquired with the 600 lines $\mathrm{mm}^{-1}$ grating, and cover the wavelength range between $5800 \AA$ and $8500 \AA$ A. The blue spectra and a detailed description of the data will be presented in a forthcoming paper (C. Scarlata et al. 2010 , in preparation). Six objects have redshifted $\mathrm{H} \beta(\mathrm{H} \alpha)$ lines outside the observed spectral range. The exposure times ranged 
Table 1

The sample.

\begin{tabular}{|c|c|c|c|c|c|c|c|}
\hline Object & $\begin{array}{l}\text { R.A. } \\
(\text { deg })\end{array}$ & $\begin{array}{l}\text { Decl. } \\
\text { (deg) }\end{array}$ & $\begin{array}{c}\text { Ly } \alpha \text { flux } \\
\operatorname{erg~cm}^{-2} s^{-1}\end{array}$ & $\begin{array}{c}\mathrm{H} \alpha \text { flux } \\
\mathrm{erg} \mathrm{cm}^{-2} \mathrm{~s}^{-1}\end{array}$ & $\begin{array}{c}\mathrm{H} \beta \text { flux } \\
\operatorname{erg~cm}^{-2} \mathrm{~s}^{-1}\end{array}$ & $z^{\mathrm{b}}$ & Class $^{\mathrm{c}}$ \\
\hline GROTH-13305 & 215.8867 & 52.6237 & $1.70 \times 10^{-15}$ & $(1.49 \pm 0.10) \times 10^{-15}$ & $(2.2 \pm 0.1) \times 10^{-16}$ & 0.2836 & SF \\
\hline GROTH-14069 & 215.3526 & 52.6555 & $1.80 \times 10^{-15}$ & $(2.58 \pm 0.10) \times 10^{-15}$ & $(7.37 \pm 0.69) \times 10^{-16}$ & 0.2582 & SF \\
\hline GROTH-17005 & 215.1805 & 52.7188 & $2.87 \times 10^{-15}$ & $(1.18 \pm 0.08) \times 10^{-15}$ & $(3.23 \pm 0.52) \times 10^{-16}$ & 0.2467 & SF \\
\hline GROTH-17525 & 215.8241 & 52.7135 & $2.62 \times 10^{-15}$ & $(2.58 \pm 0.13) \times 10^{-15}$ & $<6.15 \times 10^{-16}$ & 0.2770 & SF \\
\hline GROTH-17867 & 215.8429 & 52.7425 & $1.21 \times 10^{-15}$ & $(1.28 \pm 0.10) \times 10^{-15}$ & $(2.73 \pm 0.43) \times 10^{-16}$ & 0.2774 & SF \\
\hline GROTH-19002 & 214.4387 & 52.7719 & $2.94 \times 10^{-15}$ & $(5.77 \pm 0.58) \times 10^{-16}$ & $<3.04 \times 10^{-16}$ & 0.2439 & SF \\
\hline GROTH-36336 & 214.5818 & 53.3393 & $2.24 \times 10^{-15}$ & $(1.95 \pm 0.15) \times 10^{-15}$ & $<1.04 \times 10^{-15}$ & 0.2647 & SF \\
\hline GROTH-36896 & 214.9730 & 53.3764 & $3.29 \times 10^{-15}$ & $(2.53 \pm 0.05) \times 10^{-15}$ & $(4.46 \pm 0.50) \times 10^{-16}$ & 0.1942 & SF \\
\hline GROTH-5715 & 214.2262 & 52.4111 & $3.59 \times 10^{-15}$ & $(1.78 \pm 0.07) \times 10^{-15}$ & $(3.82 \pm 0.34) \times 10^{-16}$ & 0.2466 & SF \\
\hline GROTH-37457 & 214.7951 & 53.2660 & $4.38 \times 10^{-15}$ & $(2.43 \pm 0.12) \times 10^{-15}$ & $<8.00 \times 10^{-16}$ & 0.2639 & AGN \\
\hline GROTH-31403 & 214.2910 & 53.0867 & $2.25 \times 10^{-15}$ & $(1.10 \pm 0.06) \times 10^{-15}$ & $(2.99 \pm 0.55) \times 10^{-16}$ & 0.2672 & $\mathrm{SF}$ \\
\hline GROTH-21404 & 215.1861 & 52.8351 & $2.53 \times 10^{-15}$ & $(2.06 \pm 0.06) \times 10^{-15}$ & $(5.30 \pm 0.29) \times 10^{-16}$ & 0.2518 & $\mathrm{SF}$ \\
\hline GROTH-3525 & 214.7796 & 52.3522 & $2.41 \times 10^{-15}$ & $(1.30 \pm 0.11) \times 10^{-15}$ & $<1.79 \times 10^{-15}$ & 0.2649 & $\mathrm{AGN}^{\mathrm{d}}$ \\
\hline GROTH-37380 & 215.1904 & 53.3248 & $1.93 \times 10^{-15}$ & $(1.32 \pm 0.08) \times 10^{-15}$ & $(4.92 \pm 0.38) \times 10^{-16}$ & 0.2631 & SF \\
\hline GROTH-12279 & 214.3008 & 52.5991 & $2.08 \times 10^{-15}$ & $(1.34 \pm 0.06) \times 10^{-15}$ & $(5.02 \pm 0.43) \times 10^{-16}$ & 0.2612 & SF \\
\hline GROTH-20285 & 215.1330 & 52.7994 & $4.11 \times 10^{-15}$ & $(1.41 \pm 0.03) \times 10^{-15}$ & $(4.08 \pm 0.28) \times 10^{-16}$ & 0.2517 & SF \\
\hline GROTH-7430 & 214.4311 & 52.4683 & $7.67 \times 10^{-15}$ & $(2.03 \pm 0.08) \times 10^{-15}$ & $(6.85 \pm 0.91) \times 10^{-16}$ & 0.2079 & SF \\
\hline NGPDWS-23690 & 219.8446 & 35.3075 & $5.88 \times 10^{-15}$ & $(3.86 \pm 0.10) \times 10^{-15}$ & $(9.95 \pm 0.63) \times 10^{-16}$ & 0.2477 & SF \\
\hline NGPDWS-10002 & 219.0922 & 34.9421 & $4.33 \times 10^{-15}$ & $(2.06 \pm 0.06) \times 10^{-15}$ & $(7.52 \pm 0.44) \times 10^{-16}$ & 0.2685 & SF \\
\hline NGPDWS-33782 & 219.5770 & 35.6305 & $6.05 \times 10^{-15}$ & $(1.80 \pm 0.08) \times 10^{-15}$ & $(5.45 \pm 0.39) \times 10^{-16}$ & 0.2618 & $\mathrm{SF}$ \\
\hline NGPDWS-11927 & 219.1004 & 34.9935 & $4.34 \times 10^{-15}$ & $(7.09 \pm 0.48) \times 10^{-16}$ & $\ldots$ & 0.2145 & $\mathrm{SF}^{\mathrm{d}}$ \\
\hline NGPDWS-23216 & 218.6954 & 35.2844 & $4.09 \times 10^{-15}$ & $(3.66 \pm 0.40) \times 10^{-15}$ & $<5.74 \times 10^{-15}$ & 0.1902 & SF \\
\hline NGPDWS-6731 & 219.1529 & 34.8428 & $1.01 \times 10^{-14}$ & $(4.41 \pm 0.11) \times 10^{-15}$ & $(9.61 \pm 0.47) \times 10^{-16}$ & 0.2796 & AGN \\
\hline NGPDWS-28521 & 219.0262 & 35.4586 & $4.05 \times 10^{-15}$ & $(1.52 \pm 0.08) \times 10^{-15}$ & $(4.07 \pm 0.52) \times 10^{-16}$ & 0.2493 & $\mathrm{SF}$ \\
\hline NGPDWS-35813 & 219.0558 & 35.7291 & $3.70 \times 10^{-15}$ & $(7.41 \pm 0.47) \times 10^{-16}$ & $(2.39 \pm 0.30) \times 10^{-16}$ & 0.2596 & SF \\
\hline NGPDWS-32840 & 219.2433 & 35.5977 & $2.32 \times 10^{-15}$ & $(3.83 \pm 0.15) \times 10^{-15}$ & $<5.69 \times 10^{-16}$ & 0.2058 & $\mathrm{AGN}^{\mathrm{d}}$ \\
\hline SIRTFFL-14450 & 259.2110 & 59.9642 & $2.22 \times 10^{-14}$ & $(2.14 \pm 0.02) \times 10^{-14}$ & $(6.10 \pm 0.14) \times 10^{-15}$ & 0.1815 & SF \\
\hline SIRTFFL-14085 & 258.1492 & 59.9468 & $2.87 \times 10^{-15}$ & $(3.21 \pm 0.07) \times 10^{-15}$ & $(9.83 \pm 0.80) \times 10^{-16}$ & 0.2203 & $\mathrm{SF}$ \\
\hline SIRTFFL-10895 & 258.5918 & 59.8333 & $6.18 \times 10^{-15}$ & $(1.23 \pm 0.05) \times 10^{-15}$ & 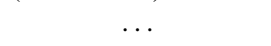 & 0.2297 & $\mathrm{SF}^{\mathrm{d}}$ \\
\hline
\end{tabular}

Notes.

${ }^{a}$ Ly $\alpha$ flux from Deharveng et al. (2008).

${ }^{b}$ Redshift computed from the optical emission lines.

${ }^{c}$ Class: galaxy classification based on the optical emission lines. SF; star-forming galaxy; AGN: active nucleus.

${ }^{\mathrm{d}}$ Classification in $\mathrm{SF}$ or AGN based on the $[\mathrm{N} \mathrm{II}] / \mathrm{H} \alpha$ ratio. GROTH-23096 was classified as an AGN based on the width of the [O III] emission lines.

between $2 \times 200 \mathrm{~s}$ and $4 \times 600 \mathrm{~s}$. During the observations seeing ranged from 1 to 1 .".5. The galaxies were observed using a $1^{\prime \prime} .5$ wide slit, providing a spectral resolution of $8 \AA$ (i.e., $280 \mathrm{~km}$ $\mathrm{s}^{-1}$ at $z=0.3$ ). This resolution is high enough to resolve the $\mathrm{H} \alpha$ from [N II] emission lines. To check for slit loss, we also observed eight galaxies with the 5".0 wide slit.

Bias subtraction, flat-field normalization, wavelength calibration, and extraction of the one-dimensional spectra were performed with standard IRAF packages. The wavelengthdependent instrumental response was removed by normalizing each spectrum with the response curve derived from the spectrum of a standard star observed in one of our photometric nights. The final flux calibration was performed using the total $i$-band magnitude of each galaxy available from the Sloan Digital Sky Survey (SDSS; York et al. 2000). We convolved each spectrum with the filter transmission curve ${ }^{10}$ and compare the resulting flux with the total SDSS flux. With this approach, the calibrated spectra are simultaneously corrected for slit losses, due to the $1^{\prime \prime} .5$ size of the slit. ${ }^{11}$ Although valid for the stellar component (i.e., the continuum) of the galaxies, if the gas is more concentrated than the stars this correction might introduce

\footnotetext{
10 The $900 \AA$ A width of the $i$ filter is fully contained within the observed red spectra.

11 The median half-light radius of the sample galaxies is $\sim 0^{\prime} 9$.
}

a systematic overestimate of the emission line intensities (without affecting the line ratios). In order to check this effect, we computed $f_{1.5} / f_{55^{\prime \prime} 0}$, i.e., the ratio between the $\mathrm{H} \alpha$ fluxes measured from the 1".5 and the 5".0 slit, for those galaxies for which the two spectra were available. On average, the line intensities agree to within $25 \%$. This potential source of error would affect the ratio between our measured optical line intensities and that of $\operatorname{Ly} \alpha$, in the direction of systematically underestimating the $\mathrm{Ly} \alpha / \mathrm{H} \alpha$ ratio. One source has a $\mathrm{Ly} \alpha / \mathrm{H} \alpha /$ ratio $>5$. Even for this object, a $25 \%$ error on the $\mathrm{H} \alpha$ would not result in a $\mathrm{Ly} \alpha / \mathrm{H} \alpha$ ratio greater than the case $B$ value.

We measured line intensities and EWs by fitting a Gaussian to the emission lines, and checked the results using the IRAF package SPLOT. All Balmer emission line intensities were corrected for stellar absorption, fitting the continuum of each spectrum between 2600 and $6600 \AA$ rest frame with synthetic templates from the Bruzual \& Charlot library (Bruzual \& Charlot 1993, 2003). Templates with constant star formation history, different age, and metallicity were employed. The median values of the stellar absorption rest-frame $\mathrm{EW}$ for $\mathrm{H} \alpha$ and $\mathrm{H} \beta$ in our galaxies are, respectively, $3.38 \AA$ and $4.18 \AA$. For an $\mathrm{EW}$ of $20 \AA$, these values correspond to a flux correction of about $21 \%$ and $27 \%$ in $\mathrm{H} \alpha$ and $\mathrm{H} \beta$, respectively. We also applied a Ly $\alpha$ stellar absorption correction of $10 \AA \mathrm{EW}$ to all galaxies 


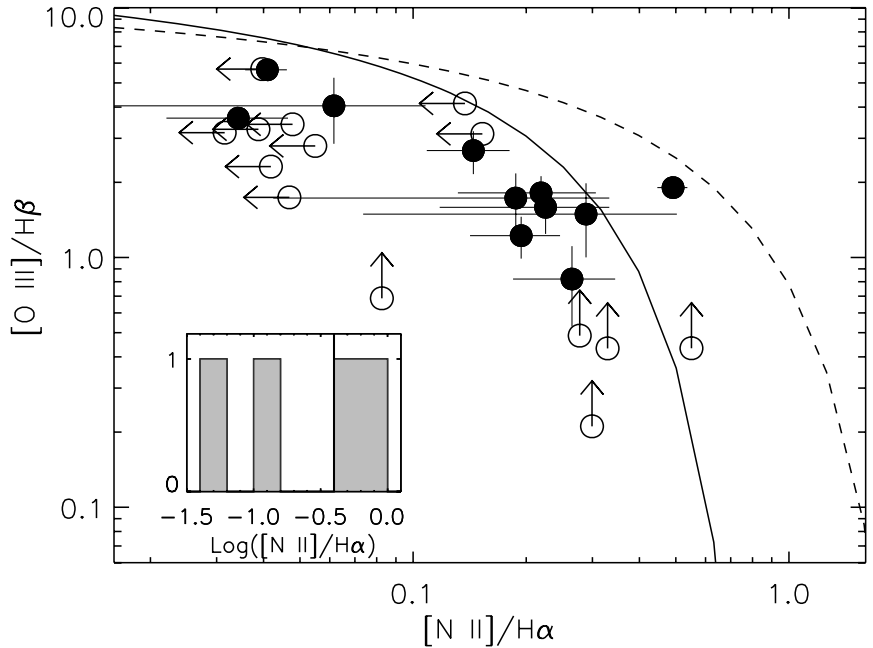

Figure 1. BPT diagnostic diagram: $[\mathrm{O} \mathrm{III}] / \mathrm{H} \beta$ is plotted against $[\mathrm{N} \mathrm{II}] / \mathrm{H} \alpha$ for the GALEX Ly $\alpha$ emitters (filled circles and limits). When a line was not detected, we assign the $3 \sigma$ upper limit to the line intensity. The dashed and solid curved lines represent the Kewley et al. (2001) and Kauffmann et al. (2003) classification lines, respectively, which divide $\mathrm{H}$ II regions (below the lines) from the region occupied by AGNs (above the lines). To be conservative, we use the Kauffmann et al. (2003) curve to identify AGNs in our sample. Inset: histogram of the $\mathrm{H} \alpha /\left[\mathrm{N}_{\mathrm{II}}\right]$ ratio for the four galaxies for which $\mathrm{H} \beta$ fell outside the wavelength range covered by the spectra. A galaxy is classified as an AGN if $\log ([\mathrm{N}$ II $/ \mathrm{H} \alpha])>-0.4$. One object, not shown in this figure, was classified as an AGN based on the width of the [O III] emission line (see the text).

with a best-fit age older than $35 \mathrm{Myr}$, following Valls-Gabaud (1993).

\section{AGN IDENTIFICATION}

Deharveng et al. (2008) identified broad-line active galactic nuclei (AGNs) in their sample of Ly $\alpha$ emitters as those objects with Ly $\alpha$ line widths broader than $1200 \mathrm{~km} \mathrm{~s}^{-1}$. Narrow-line AGNs could not be identified since other diagnostic lines of AGN activity were either too faint, or fell in a noisy part of the UV spectra. In order to identify the narrow-line AGN, we have used the classic Baldwin et al. (1981, BPT) diagram of $[\mathrm{O}$ III] $/ \mathrm{H} \beta$ versus $[\mathrm{N}$ II] $/ \mathrm{H} \alpha$ (as shown in Figure 1 ).

The theoretical maximum line ratios possible for pure stellar photoionization are shown as a dashed curve in Figure 1 (Kewley et al. 2001). To be conservative, we use the Kauffmann et al. (2003) empirical separation between active and star-forming galaxies (solid line). We find that in two of our Ly $\alpha$ emitters the gas is likely to be ionized by an active nucleus. We note that both cases are in the region of the diagram where stellar photoionization cannot be excluded on the basis of theoretical calculations (Kewley et al. 2001). Among the six galaxies that could not be placed on the BPT diagram, we could still classify five of them using either the $[\mathrm{N}$ II $] / \mathrm{H} \alpha$ ratio, or the line width. We identify the AGNs as those galaxies for which $\log ([\mathrm{N} \mathrm{II}] / \mathrm{H} \alpha) \geqslant-0.4$ (Miller et al. 2003; Carter et al. 2001). We add two AGNs based on the $[\mathrm{N}$ II $] / \mathrm{H} \alpha$ ratio. Finally, one of the two galaxies for which the $\mathrm{H} \alpha$ is too red to be covered by our spectrum has an [O III] line width typical of an AGN $\left(\sim 1090 \mathrm{~km} \mathrm{~s}^{-1}\right)$. To summarize, we identify five AGNs out of a sample of 30 galaxies, i.e, the fraction of Ly $\alpha$ emitters classified as AGNs is $17 \%$, consistent with the recent measurement by Cowie et al. $(2009,15 \%)$, and marginally consistent with the measurement by Finkelstein et al. (2009b, 43-26 \%). However, Finkelstein et al. (2009b) classify AGNs using also the presence of high-ionization emission lines, that may be indicative of a high-excitation star forming galaxy, rather than an active nucleus.

In the following analysis, we only consider those 20 galaxies that are classified as star forming, and with both $\mathrm{H} \alpha$ and $\mathrm{H} \beta$ detected in the spectrum.

\section{DUST GEOMETRY AND REDDENING}

In the absence of scattering, the EW of a line is by definition unaffected by dust extinction. For this reason, the Ly $\alpha$ EW has often been used to study the effect of scattering on the Ly $\alpha$ photons, by comparing the observed values with those expected theoretically (e.g., Giavalisco et al. 1996). The intrinsic Ly $\alpha$ EW is, however, predicted to vary by more than 2 orders of magnitude within the first $10 \mathrm{Myr}$ of a starburst (Charlot \& Fall 1993), and depends on the IMF of the stellar population.

The observed line intensities depend only on the number of ionizing photons and the attenuation produced by dust along the line of sight. Compared to EW, line ratios are a more direct probe of the obscuration of the line photons. For this reason, the comparison of the ratios of resonant to non-resonant lines (i.e., $\mathrm{Ly} \alpha / \mathrm{H} \alpha)$ with the ratio of two non-resonant lines $(\mathrm{H} \alpha / \mathrm{H} \beta)$ is optimal for quantifying the effects of dust extinction and resonant scattering on the escape of Ly $\alpha$ photons.

In Figure 2, we show the $\mathrm{Ly} \alpha / \mathrm{H} \alpha$ ratio as a function of the observed $\mathrm{H} \alpha / \mathrm{H} \beta$ ratio. Both quantities are corrected for stellar absorption. In the absence of dust, for case B recombination, $T_{e} \sim 10^{4} \mathrm{~K}$, and $n_{e}=10^{2} \mathrm{~cm}^{-3}$, the expected line ratios are 2.86 and 8.7 for $\mathrm{H} \alpha / \mathrm{H} \beta$ and $\mathrm{Ly} \alpha / \mathrm{H} \alpha$, respectively (Pengelly 1964). The galaxies show a broad range of $\mathrm{H} \alpha / \mathrm{H} \beta$ values going from the value predicted in case B recombination theory up to an $\mathrm{H} \alpha / \mathrm{H} \beta \sim 7$. Apart from one galaxy, the $\mathrm{Ly} \alpha / \mathrm{H} \alpha$ ratio is always smaller than $50 \%$ of the value predicted for case $\mathrm{B}$.

In order to interpret the observed line ratios, we will first ignore the effects of resonant scattering on the Ly $\alpha$ photons, and consider only the effect of the dust extinction (derived using the $\mathrm{H} \alpha / \mathrm{H} \beta$ ratio) on the $\mathrm{Ly} \alpha / \mathrm{H} \alpha$ ratio. Traditionally, this analysis is carried out under the simplifying assumption that the dust is distributed uniformly in a screen far from the source (e.g., Giavalisco et al. 1996; Hayes et al. 2007; Atek et al. 2008; Finkelstein et al. 2008, 2009a). Although valid for pointlike sources, this simple approximation is found not to hold in more complex systems, as shown by the analysis of Galactic and extragalactic $\mathrm{H}$ II regions, where the uniform dust screen approximation fails to reproduce the observed Balmer line ratios (e.g., Natta \& Panagia 1984; Caplan \& Deharveng 1986). More complex geometries do predict different attenuation laws, with the net effect of removing the linearity between the dust optical depth and the logarithm of the observed line intensity.

In order to illustrate this effect, we consider here three different geometries of the dust around the line-emitting regions: (1) uniform dust screen in front of the emitting sources; (2) dust distributed in clumps; (3) sources uniformly mixed within uniform dust. The light attenuation ${ }^{12}$ has a different behavior in the three cases. In model (1), the attenuation is given by the classical $\exp \left(-\tau_{\lambda}\right)$, where the optical depth $\tau_{\lambda}$ follows the appropriate extinction curve, and depends only on the intrinsic grain properties. We assume that the dust/gas relative geometry does not affect the physical properties of the grains, and we adopt the extinction curve as parameterized by Cardelli et al. $\left(1989, \tau_{\lambda} / \tau_{V}\right)$. In model (2), Natta \& Panagia (1984) calculate

12 The attenuation is defined as $I_{\lambda} / I_{\lambda}^{0}$, where $I_{\lambda}$ and $I_{\lambda}^{0}$ are the observed and intrinsic intensities at $\lambda$. 


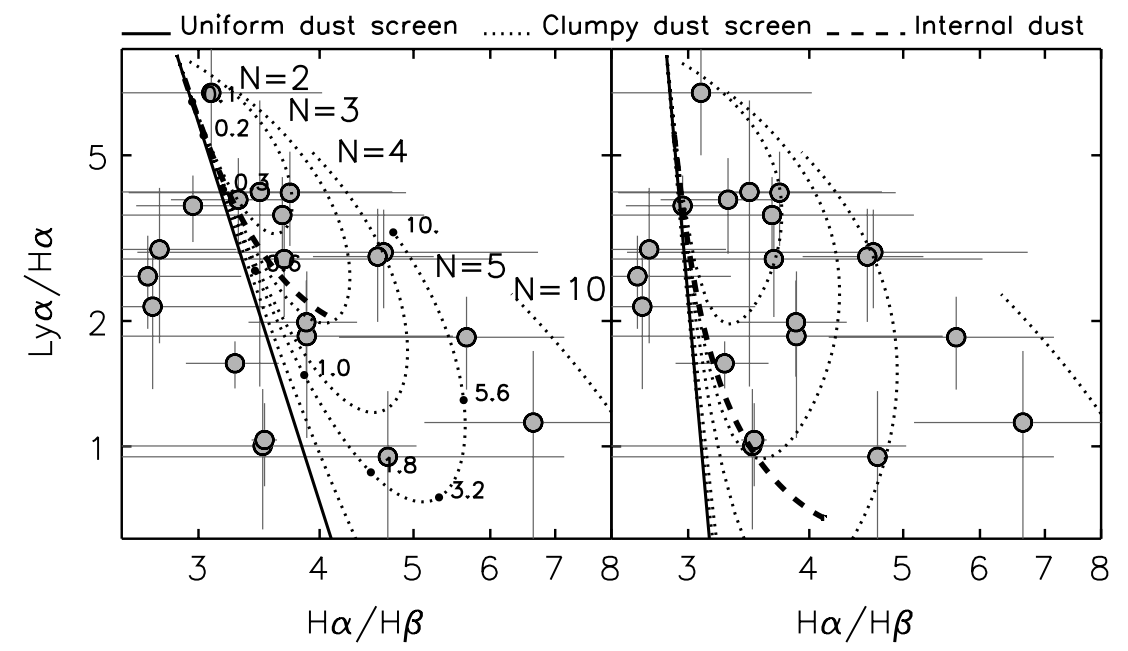

Figure 2. $\mathrm{Ly} \alpha / \mathrm{H} \alpha$ ratio against $\mathrm{H} \alpha / \mathrm{H} \beta$ ratio: the various curves show the behavior of the line ratios as a function of increasing optical depth of the dust, assuming three different geometries for the dust/source distribution: uniform dust screen (solid line), clumpy distribution (dotted lines) for different values of the average numbers of clumps, $N$, along the line of sight, and internal dust model (dashed line). The numbers near the black dots on the curve for the clumpy medium with $N=5$ show $\tau_{V}$ at that position. In the left panel, all models are computed assuming $\tau_{\mathrm{Ly} \alpha}=\tau_{1216}$, while in the right panel we account for the scattering of the Ly $\alpha$ photons assuming $\tau_{\mathrm{Ly} \alpha}=3 \times \tau_{1216}$ (see the text for details).

the attenuation assuming that the dust clumps are Poissonian distributed, and that all clumps have the same optical depth, $\tau_{c, \lambda}$. Within each clump, $\tau_{c, \lambda}$ follows the Cardelli et al. (1989) extinction law. If $N$ is the average number of clumps along the line of sight, then they find that $I_{\lambda} / I_{\lambda}^{0}=\exp \left[-N\left(1-e^{-\tau_{c, \lambda}}\right)\right]$. For a large average number of clumps, and a moderate value of $\tau_{V}$ for each clump, the attenuation tends to the uniform dust screen. However, the two models differ greatly if the clumps have large optical depth. In the limit of opaque clumps, the attenuation tends toward the constant value of $e^{-N}$, i.e., the extinction becomes independent of wavelength, because only the unextinguished light can be seen. In the third case considered, $I_{\lambda} / I_{\lambda}^{0}$ goes as $\left(1-e^{-\tau_{\lambda}}\right) / \tau_{\lambda}$ (Mathis 1972). In this model for $\tau_{\lambda} \rightarrow \infty$, the attenuation reaches the asymptotic value of $\tau_{\lambda}^{-1}$. Curves corresponding to the three models are shown in Figure 2 with different line styles as indicated on top of the figure. We use $\tau_{V}$ to parameterize the observed line ratios in each of the three models. The black dots along the curve of the clumpy distribution with $N=5$ are labeled with the optical depth $\tau_{V}$ at that position.

In reality, Ly $\alpha$ photons will be scattered within the clumps, effectively enhancing their chances of being absorbed by dust. This effect can be folded in the model by assuming that the optical depth for Ly $\alpha$ photons, $\tau_{\mathrm{Ly} \alpha}$, is higher than the corresponding continuum optical depth, i.e., $\tau_{\text {Ly } \alpha}=s \times \tau_{1216}$. The right panel of Figure 2 shows the same models described above, but computed accounting for the scattering of the Ly $\alpha$ photons, assuming, as an example, $s=3$. As expected, the net effect of increasing the optical depth of the Ly $\alpha$ photons results in lower values of the $\mathrm{Ly} \alpha / \mathrm{H} \alpha$ ratios for small optical depth. However, for high values of the optical depth, the asymptotic behavior of the curves remains unchanged, and so do our conclusions.

\section{DISCUSSION}

From Figure 2, we can see that at $\mathrm{H} \alpha / \mathrm{H} \beta \sim 4$ the uniform dust screen model (solid line) predicts an observed Ly $\alpha / \mathrm{H} \alpha$ ratio of $\lesssim 10 \%$ of the case $\mathrm{B}$ value. Clearly, the observed values of $\mathrm{Ly} \alpha / \mathrm{H} \alpha$ for $\mathrm{H} \alpha / \mathrm{H} \beta \geqslant 4$ are much higher than the prediction of a simple dust screen. Correcting the Ly $\alpha$ luminosities using the attenuation derived for a uniform dust screen would result in a corrected line ratio-hereafter ( $\operatorname{Ly} \alpha /$ $\mathrm{H} \alpha)_{C}$-well above the predicted value of 8.7. Neufeld (1991) and Hansen et al. (2006) discuss a multiphase geometry, where the dust is confined in cold clumps and immersed in ionized gas. A topology of this kind could serve to shield the Ly $\alpha$ photons from the effect of the dust absorption, because resonant photons would be scattered on the surface of the clumps, without crossing them. The Balmer and continuum photons, not affected by the presence of the neutral Hydrogen, would pass through the clumps, leading to a relative enhancement of the Ly $\alpha \mathrm{EW}$ and $\mathrm{Ly} \alpha /$ Balmer line ratios. Given the stochastic nature of the Ly $\alpha$ scattering, this model should also result in some objects with uncorrected $\mathrm{Ly} \alpha / \mathrm{H} \alpha$ greater than the predicted case B value. As noted above, we do not observe any galaxy with Ly $\alpha$ / $\mathrm{H} \alpha>8.7$. Furthermore, if the continuum is affected by the dust while the Ly $\alpha$ photons are not, then this should result in a correlation between the Ly $\alpha$ EW and the observed $\mathrm{H} \alpha / \mathrm{H} \beta$ ratio. In fact the four most extreme cases (with $\mathrm{Ly} \alpha / \mathrm{H} \alpha$ ratios more than 10 times higher the dust screen value) have among the lowest EW.

A model with the dust uniformly mixed with the sources is not able to reproduce the galaxies with high line ratios (the dashed line in Figure 2). This is due to the asymptotic behavior of the attenuation curve for large optical depth. While in a uniform dust screen the $\mathrm{H} \alpha / \mathrm{H} \beta$ (and $\mathrm{Ly} \alpha / \mathrm{H} \alpha$ ) can reach arbitrarily high (low) values, in the uniformly mixed model the observed $\mathrm{H} \alpha / \mathrm{H} \beta$ ratio reaches the asymptotic value of $2.86 \times \tau(\mathrm{H} \beta) / \tau(\mathrm{H} \alpha)=4.15$, and similarly a $\mathrm{Ly} \alpha / \mathrm{H} \alpha$ of $8.7 \times \tau(\mathrm{H} \alpha) / \tau(\mathrm{Ly} \alpha)=2.1$. In this model, the light that is able to reach the observer must come through a thin superficial shell of the galaxy.

Figure 2 demonstrates that the clumpy dust screen can explain the observed data. By varying the average number of clumps along the line of sight, and the intrinsic optical depth of the clumps, the clumpy distribution of dust allows $\mathrm{H} \alpha / \mathrm{H} \beta$ ratios as high as 6 while still allowing a $\mathrm{Ly} \alpha / \mathrm{H} \alpha$ ratio of $\sim 2$. This is due to the fact that for high values of $\tau_{V}$ only the fraction of light that is traveling along sight lines relatively clear of clumps is able to escape the galaxy. We consider an average number of 
clumps $N$ along the line of sight in the range from 2 to 10 . It is reasonable to expect that galaxies may have different physical structures, depending on the orientation and size of the galaxies, and that they can be statistically described by different values of $N$.

For three galaxies, the $\mathrm{H} \alpha / \mathrm{H} \beta$ ratio is consistent with 2.86 (case B), while the $\mathrm{Ly} \alpha / \mathrm{H} \alpha$ ratios are $\sim 70 \%$ lower than that case B would predict. Such galaxies require an additional mechanism to reduce Ly $\alpha$ without affecting the Balmer photons. Possibly, the Ly $\alpha$ photons pass through a neutral Hydrogen cloud, without scattering on its surface. This would not alter the observed Balmer line ratios but could suppress part of the Ly $\alpha$ photons. Kinematically resolved spectra might test this hypothesis, depending on the kinematics of the $\mathrm{H}$ I with respect to the $\mathrm{H}$ in region.

\section{CONCLUSIONS}

We have presented the analysis of optical spectra of a sample of $31 z \sim 0.3$ Ly $\alpha$ emitters, previously identified by Deharveng et al. (2008). We find that in $17 \%$ of the Ly $\alpha$ emitters the line ratios require the hard ionizing continuum produced by an AGN. This fraction is in agreement with the fraction of AGN discovered in local samples of emission line galaxies, covering a similar range of luminosities.

A uniform dust screen is not able to simultaneously reproduce the observed high values of $\mathrm{Ly} \alpha / \mathrm{H} \alpha$ and $\mathrm{H} \alpha / \mathrm{H} \beta$ line ratios. Traditionally, these are explained with ad hoc multiphase models in which Ly $\alpha$ photons are shielded from the dust, while nonresonant photons are not (e.g., Neufeld 1991). We consider alternative possibilities for the geometry of the dust around the emitting sources and find that the observed line ratios are well reproduced by a clumpy dust distribution. We cannot rule out the possibility that a combination of dusty and uniform medium is responsible for the observed line ratios. Our analysis shows that there is no need to invoke models requiring a significant percentage of $\operatorname{Ly} \alpha$ photons to escape from the galaxy through resonant scattering that avoids the dust. Furthermore, the assumption of a uniform dust screen would result in an overcorrection of the Ly $\alpha$ intensity.

\section{REFERENCES}

Atek, H., Kunth, D., Hayes, M., Ostlin, G., \& Mas-Hesse, J. M. 2008, A\&A, 488, 491

Atek, H., et al. 2009, A\&A, in press (arXiv:0906.5349v2)

Baldwin, J. A., Phillips, M. M., \& Terlevich, R. 1981, PASP, 93, 5

Bruzual, A. G., \& Charlot, S. 1993, ApJ, 405, 538

Bruzual, G., \& Charlot, S. 2003, MNRAS, 344, 1000

Caplan, J., \& Deharveng, L. 1986, A\&A, 155, 297

Cardelli, J. A., Clayton, G. C., \& Mathis, J. S. 1989, ApJ, 345, 245

Carter, B. J., Fabricant, D. G., Geller, M. J., Kurtz, M. J., \& McLean, B. 2001, ApJ, 559, 606

Charlot, S., \& Fall, S. M. 1993, ApJ, 415, 580

Cowie, L. L., Barger, A. J., \& Hu, E. M. 2009, arXiv:0909.0031

Deharveng, J.-M., et al. 2008, ApJ, 680, 1072

Dijkstra, M., Haiman, Z., \& Spaans, M. 2006, ApJ, 649, 14

Finkelstein, S. L., et al. 2008, ApJ, 678, 655

Finkelstein, S. L., et al. 2009a, ApJ, 700, 276

Finkelstein, S. L., et al. 2009b, ApJ, 703, L162

Giavalisco, M., Koratkar, A., \& Calzetti, D. 1996, ApJ, 466, 831

Hansen, M., \& Oh, S. P. 2006, MNRAS, 367, 979

Hayes, M., et al. 2007, MNRAS, 382, 1465

Kauffmann, G., et al. 2003, MNRAS, 341, 33

Kewley, L. J., et al. 2001, ApJ, 556, 121

Laursen, P., \& Sommer-Larsen, J. 2007, ApJ, 657, L69

Mathis, J. S. 1972, ApJ, 176, 651

Miller, C. J., et al. 2003, ApJ, 597, 142

Natta, A., \& Panagia, N. 1984, ApJ, 287, 228

Neufeld, D. A. 1990, ApJ, 350, 216

Neufeld, D. A. 1991, ApJ, 370, L85

Oke, J. B., \& Gunn, J. E. 1982, PASP, 94, 586

Ostlin, G., Hayes, M., Kunth, D., Mas-Hesse, J. M., Leitherer, C., Petrosian, A., \& Atek, H. 2009, AJ, 138, 923

Panagia, N., \& Ranieri, M. 1973a, A\&A, 24, 219

Panagia, N., \& Ranieri, M. 1973b, Les Nebuleuses Planétaires, 275

Pengelly, R. M. 1964, MNRAS, 127, 145

Valls-Gabaud, D. 1993, ApJ, 419, 7

Verhamme, A., Schaerer, D., \& Maselli, A. 2006, A\&A, 460, 397

York, D. G., et al. 2000, AJ, 120, 1579 